extended to other cases and can be developed into a powerful tool for examining permanent currents as well as changes produced by changing winds. Efforts in this direction are being continued.

* Contributions from the Scripps Institution of Oceanography, New Series, No. 324.

1 Ekman, V. W., Annalen Hydrographie u. Mar. Met., 34, 423-430 (1906).

${ }^{2}$ Stockmann, W., Comptes rendus (Doklady) l'Acad. sci. l'U.R.S.S., 52, 309-312 (1946).

${ }^{3}$ Fjeldstad, J. E., Archiv Math. Naturvid., 48, no. 6 (1946).

${ }^{4}$ Montgomery, R. B., and Palmén, E., Jour. Marine Research, 3, 112-133 (1940).

5 Ekman, V. W., Gerlands Beitr. z. Geophysik, Suppl. 4 (1939).

'Defant, A., Deutsche Atlantische Exped. "Meteor" 1925-27, Wiss. Ergebn., 4, no. 2, 191-260 (1941).

' Fleming, J. A., et al., Sci. Results Cruise VII "Carnegie" 1928-29, I-B (1945).

${ }^{8}$ Sverdrup, H. U., and Staff, Records Observations, Scripps Institution of Oceanography, 1, 65-160 (1943).

' U. S. Weather Bureau, W. B. No. 1247 (1938).

${ }^{10}$ Rossby, C.-G., Papers Phys. Oceanography Meteorology, 4, no. 3 (1936).

\title{
THE PROBLEMS OF CONGRUENT NUMBERS AND CONCORDANT FORMS
}

By E. T. Bell

\section{California Institute of Technology, Pasadena}

Communicated August 12, 1947

1. Four Related Problems.-All letters in formulas denote rational integers, and solution means the complete solution in such integers. The problem of solving the simultaneous diophantine equations

$$
r X^{2}+m Y^{2}=r Z^{2}, \quad s X^{2}+n Y^{2}=s W^{2}
$$

includes as special cases two classical problems.

Problem 1.-If $r=s=Y^{2}=1, n=-m$, where $m$ is a given constant, the problem is that of congruent numbers. It goes back to Diophantus in the third century, the Arabs of the tenth and eleventh centuries, and Leonardo of Pisa (Fibonacci) in the early thirteenth century. For $m$ arbitrarily assigned it is still unsolved.

Problem 2.-For $r=s=1$ the problem is Euler's (1780) of concordant forms, also unsolved.

Many special cases of these two have been investigated. Thus Fermat proved by his method of descent that if $m=n=-1$ in Problem 2, there are no integers $X, Y, Z, W$ all different from zero satisfying the equations. From this his theorem for fourth powers follows. Modern work originating in these problems has been concerned with cubics and quartics having 
at most a finite number of sets of values of the indeterminates satisfying the equations. Some of this has used the theory of the units in special algebraic number rings. From the results it is possible, by the method applied to Problem 3, to derive much information on new diophantine systems of degrees higher than the second. This will be considered elsewhere. For the present, the inherent complexity of the solution of Problem 3 may suggest why these two old and apparently simple problems are still not completely solved.

Problem 3.- To state necessary and sufficient forms of $r, m, s, n$ in order that there shall exist $X, Y, Z, W$ all different from zero satisfying the equations.

A special case that has been frequently discussed may be noted. In Problem 1, the required form of $m$ is given by

$$
4 m=x y z^{2} w^{2}\left(x^{2}-y^{2}\right), w(x+y) \text { even. }
$$

The corresponding $X, Z, W$ are given by

$$
4 X=z w\left(x^{2}+y^{2}\right), 4 Z=z w\left(x^{2}+2 x y-y^{2}\right), 4 W=z w\left(x^{2}-y^{2}\right) .
$$

For $m$ squarefree, $z w= \pm 1$, giving a known criterion. The proof is immediate by the method used for solving Problem 3. Although it is not included in Problem 3, another, somewhat similar problem, dating from the Arabs and usually included with questions on congruent numbers is

Problem 4.-To state a necessary and sufficient form of $n$ in order that $X, Y, Z$ all different from zero shall exist satisfying

$$
n+X^{2}=Y^{2}, n-X^{2}=Z^{2} \text {. }
$$

The solution is given by

$$
4 n=x^{2}\left(a^{2} y^{4}+b^{2} z^{4}\right), a b=2 ;
$$

the corresponding $X, Y, Z$ are given by

$$
X=x y z, 2 Y=x\left(a y^{2}+b z^{2}\right), 2 Z=x\left(a y^{2}-b z^{2}\right) .
$$

This is equivalent to

$$
\begin{gathered}
a b=2, f g h^{2}=4, a^{2} y^{4}+b^{2} z^{4}=f u, n=g u v^{2} ; \\
X=g h y z v, 2 Y=g h v\left(a y^{2}+b z^{2}\right), 2 Z=g h v\left(a y^{2}-b z^{2}\right) .
\end{gathered}
$$

2. Solution of Problem 3.-If $r, m, s, n, X, Y, Z, W$ are indeterminates, the equations are homogeneous cubics, each of which is separable and hence (completely) solvable. The result of equating the parametric expressions for $X$ and those for $Y$ in the solutions gives a separable and hence (completely) solvable system. As the solution of separable equations, 
or of a system of such equations, is now straightforward routine, it will suffice to state the final result. To condense the formulas, write

$$
\begin{gathered}
a \equiv a_{1} a_{2} a_{3} a_{4} a_{5}, b \equiv b_{1} b_{2} b_{3} b_{4} b_{5}, c \equiv c_{1} c_{2} c_{3} c_{4} c_{5}, \\
f \equiv f_{1} f_{2} f_{3} f_{4} f_{5}, g \equiv g_{1} g_{2} g_{3} g_{4} g_{5}, h \equiv h_{1} h_{2} h_{3} h_{4} h_{5}, \\
\alpha \equiv b_{1} c_{1} f_{1} g_{1} h_{1}, \beta \equiv a_{1} c_{2} f_{2} g_{2} h_{2}, \gamma \equiv a_{2} b_{2} f_{3} g_{3} h_{3}, \\
\theta \equiv a_{3} b_{3} c_{3} g_{4} h_{4} ; \phi \equiv a_{4} b_{4} c_{4} f_{4} h_{5}, \psi \equiv a_{5} b_{5} c_{5} f_{5} g_{5} \\
\pi \equiv a b c g h, m \equiv m_{1} m_{2}, n \equiv t n_{1} h_{2} .
\end{gathered}
$$

Thus $p, m_{1}, m_{2}$ are bound parameters whose product is $m$; similarly for $t, n_{1}, n_{2}$ and $n$. The $a_{i}, \ldots, g_{i}$ are independent parameters. Define

$$
\begin{gathered}
A \equiv m_{1} a f g^{2}-n_{1} \alpha \theta \phi^{2}, B \equiv m_{2} b f h^{2}-n_{2} \beta \theta \psi^{2}, \\
C \equiv m_{2} n_{1} b h^{2} \alpha \phi^{2}-m_{1} n_{2} a g^{2} \beta \psi^{2},
\end{gathered}
$$

introduce the parameters $x, y, z$ and define $e$, for assigned values of all the parameters, as an arbitrary integer multiple of the reciprocal of the greatest common divisor of

$$
x y^{2} A, x z^{2} B, y^{2} z^{2} C \text {. }
$$

(If $e$ is merely an arbitrary integer, the values of $r, s, X, Y, Z, W$ stated presently, with $p, m_{1}, m_{2}, t, n_{1}, n_{2}$ as above, satisfy the equations identically, but this does not exhaust the possibilities. The stated definition of $e$ is necessary.) Introduce the parameter $u$. The required values of $r, s$ are

$$
r=e^{2} p u^{2} x^{2} y^{2} z^{2} a b c^{2} A B, s=e^{2} t u^{2} x^{2} y^{2} z^{2} \alpha \beta \gamma^{2} A B .
$$

To state the corresponding values of $X, Y, Z, W$ define

$$
\begin{gathered}
F \equiv m_{2} n_{1} b h^{2} \alpha \phi^{2}-a g^{2} \beta \psi^{2} \\
G \equiv 2 m_{1} m_{2} a b f g^{2} h^{2}-m_{1} n_{2} a g^{2} \beta \theta \psi^{2}-m_{2} n_{1} b h^{2} \alpha \theta \phi^{2}, \\
H \equiv m_{1} n_{2} a f g^{2} \beta \psi^{2}+m_{2} n_{1} b f h^{2} \alpha \phi^{2}-2 n_{1} n_{2} \alpha \beta \theta \phi^{2} \psi^{2} .
\end{gathered}
$$

Introduce a parameter $k$. Then

$$
\begin{gathered}
2 X=e k x^{2} y^{2} z^{2} f \theta F, Y=e^{2} k u x^{2} y^{2} z^{2} \pi A B \\
2 Z=e k x^{2} y^{2} z^{2} f G, 2 W=e k x^{2} y^{2} z^{2} \theta H
\end{gathered}
$$

Including the bound parameters $p, m_{1}, m_{2}, t, n_{1}, n_{2}$ there are in all 41 . Each of $m, n$ is of degree 3 ; each of $r, s$, is of degree 71 ; each of $X, Z, W$ is of degree 49 , and $Y$ is of degree 83 . The degree of each of the identities giving the solution of the cubic system is thus 169 . 